\title{
PLC Based Instrumentation of Closed Loop Gas System for RPC Detectors
}

\author{
S. D. Kalmani ${ }^{\mathrm{a}^{*}}$, A.V. Joshi ${ }^{\mathrm{b}}$, R.R. Shinde ${ }^{\mathrm{a}}$, and P. V. Hunagund ${ }^{\mathrm{c}}$ \\ ${ }^{a}$ Tata Institute of Fundamental Research, Dr. Homi Bhabha Road, Colaba, Mumbai, India \\ ${ }^{b}$ AlphaPneumatics, Thane (Mumbai), India \\ ${ }^{\mathrm{c}}$ Department of Applied Electronics and Research, Gulbarga University, Gulbarga, India \\ *kalmani51@gmail.com; *alpha_pneumatics@hotmail.com
}

\begin{abstract}
The INO experiment is a mega-science project and will have RPC detectors as an active element. It will be instrumented with about 28,800 RPC detectors. These active detectors use a gas mixture of R134a $(95.2 \%)$, Isobutane (4.5\%) and $S F_{6}(0.3 \%)$. Due to large number of RPC detectors, the total gas required is of the order of $200 \mathrm{M}^{3}$; therefore a Closed Loop gas recirculation System (CLS) is mandatory. The prototype CLS system for 12 such RPCs has been developed using PLC (Programmable Logical Controllers) and associated peripheral input and output devices. This CLS is based on pneumatic parameters; hence many pressure sensors are used in process control and operation. Several simple CLS are developed by the team of Alpha pneumatics. The paper high lights the instrumentation of the CLS, which is in operation at TIFR and a few results are presented.
\end{abstract}

Keywords: INO, PLC, Gas systems, Particle tracking detectors, Resistive-plate chambers, SCCM

\section{Introduction}

India-based Neutrino Observatory (INO) [1], one of the mega science project plans to build $50 \mathrm{~K}$ tons Iron Calorimeter (ICAL) to study the properties of atmospheric neutrinos particles. The experiment will use about 28,800 RPCs(gaseous detectors) with glass as electrode. Each of these detectors will have 128 readout channels on X-side and similar number on the Y-side. Therefore, 3.6 million channels need to read and processed based on criteria of trigger for neutrino studies. The RPC being a gaseous detector and its performance dependence on the quality of the gases and more over the number of these detectors are huge and considering the safety aspects, an automated CLS that will a supply mixed gas and purify it after flowing through the RPC in a loop is designed, developed and is in operation for the few years. The RPCs operate at a low pressure of about 3 mbar, with a flow rate of 6 Standard Cubic Centimeter per Minute (SCCM). In the design of CLS, the pressure parameters play a crucial role. The basic block diagram of CLS is shown in Figure 1. The CLS is completely automated and has provision to mix four type of gases (only three used) in the appropriate ratio as required by the user, typically R134a (95.2\%), Isobutene (4.5\%) and Sulphur hexafluoride $(0.3 \%)$ with a set flow rate of $6 \mathrm{SCCM}$ to each detector and maintain a safe pressure of 3 mbar for the operation of the RPCs. It contains the purification process in the loop and is automated by using dual purifiers. The safety aspects like over pressure, and isolation or cutoff of flow of gas into the RPC detector stack due to blockage of gas is implemented.

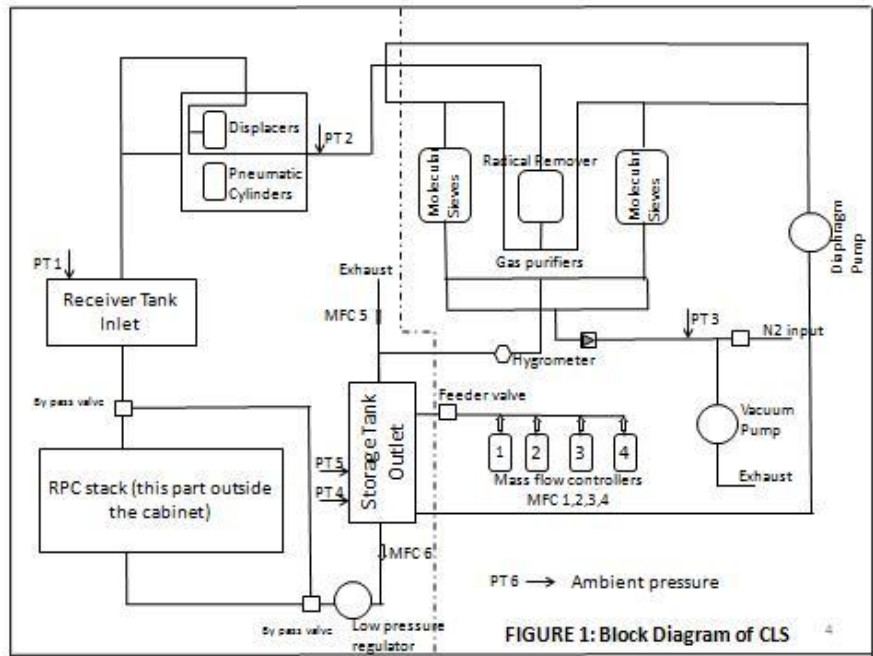




\section{Design Criteria}

\section{Principal of operation}

There are two basic pressures, PT1 measured on the receiver tank pressure and PT5 on the storage or delivery tank pressure. The PT1 is lowest in the loop while PT5 is highest. The suction pump which is a reciprocating type of pump which sucks gas from receiver tank and delivers it to storage tank. The storage acts like a buffer tank. The reciprocating pump is used for better discharge rate control and its suction is not continuous as it would have been in case of a rotary or centrifugal pump. The pressure PT1 reduces when the suction stroke is on and increases due to return flow when suction stroke is off. The PT1 being the lowest pressure in the loop and it can be assumed that the pressure inside RPC is more than PT1 and is allowed to change within a band of pressures which is usually 3 mbar. This means pressure inside RPC cannot change more than 3 mbar. The lower limiting value is termed as SP1 which is in the range of 0.998 bars to 1.003 bars. The upper pressure range is termed as SP2 and can be set at 1.000 bar to 1.006 bar depending upon the allowable pressure band. When pressure falls below SP1, the pumping stops and further lowering of PT1 does not take place. In this situation the flow is full as per set point on the MFC 6 (Mass Flow Controller) which supplies the gas into the RPC stack with the set flow rate of 6 SCCM. Due to this action PT1 tends to rise. When pressure goes above or equals SP2, the flow stops completely through MFC 6 hence PT1 cannot further increase. The pumping is kept on so that it helps in lowering PT1 to a pressure below SP2.

The flow is proportional to the Band (SP2-SP1) and actual value of PT1 is obtained in following way

Flow rate $=$ MFC6 $*((\mathrm{SP} 2-\mathrm{SP} 1) / \mathrm{PT} 1))$

The storage tank pressure (PT5) is monitored to estimate the leak rate and the amount of gas available for recirculation. The limit are set at PT5 and should not be less than 1.150 bar and more than 1.450 bar. This difference in the limit of 300 mbar is constant and internally set. The lower pressure range is termed as PBS and is adjustable. When PT5 reaches a value of 1.150 bar due to leakage or bleed through MFC 5, the backfill is activated .The selected valves which are not shown in drawing namely SV10 ( isobutene) SV11 ( Freon) and $\mathrm{SV} 12\left(\mathrm{SF}_{6}\right)$ are opened along with SV13 which is a discharge valve. When the pressure PT5 reaches PBS +300 mbar i.e. 1.450 mbar, the backfill stops. All along the backfill, the normal function of pumping and PT1 is ON along with all interlocking actions. The pump is a reciprocating piston type and driven by a pneumatic cylinder. The stroke is $100 \mathrm{~mm}$ and its bore is $18 \mathrm{~mm}$. The forward and return stroke lengths can be adjusted by the position of reed switches provided on the body of pump. The forward and reverse speeds also can be controlled by unidirectional needle valves which are provided on compressed air tubes. The supply pressure of 5 bars is required to operate the pumping. To keep the flow uniform throughout the pumping cycle and similarly the pressure should also be kept constant. A low pressure regulator is installed in the loop to reduce pressure from PT5 (1.45 bar) to an adjustable 20 to 30 mbar constant pressure (safe operation of RPCs). But 20 mbar to 30 mbar pressure is too low for MFC 6 to work on downstream of low pressure regulator. Hence we have to keep it on the upstream side of LP (Low Pressure) regulator. During a condition PT5>SP2 MFC 6 flow stops completely and this action starves LP regulator of gas sending it into unstable control. During evacuation of the system the entire volume comes under vacuum, including low pressure regulator. As this is a large area device, vacuum produces tremendous forces on the diaphragm which might get ruptured or may lose smoothness of pressure control. The storage tank delivers low pressure gas to the RPC which is collected in the receiver tank and pumped and returned to the storage tank. The fresh mixed gas is prepared through a MFC set at appropriate flow rate. The system operates between 1.15 bars to 1.45 bar where the top up starts at 1.15 bars and stops at 1.45 bars. The RPC is maintained between 1.006 to 1.009 bars with 3 mbar ratio differences. The typical flow rates can be between 5 SCCM to 100 SCCM adjustable. The room pressure variation seen is 1.004 to 1.010 bar and twice a day. The contamination like water vapor is removed by using molecular sieve type 5A and hydrocarbons (oil vapor, while decanting, RPC making etc.) by 13X. The radicals are not present in large quantities under cosmic operations however anhydrous activated alumina is used to fix (radical fragments) and trap them permanently.

\section{Instrumentation of CLS}

The key components of the CLS are PLC units namely the master controller CPU (model SIMATICS7-1214C) Figure 2a, digital input (SM 1222-DC), digital input and out module (SM 1223-DC/DC), analog input modules (SM 1231 AI) and analog output module (SM 1232 AQ), Shavison make, AS 775,AS 773,AS 333,TAON-30s/60S, TATP-180SAS 622 namely solenoid valve controller, solenoid drivers, power distribution modules, timers, analog timers, isolated coils, respectively, Schinder make relays (24V) MCBs, GE Pan metrics transmitter, Tylan make MFCs (Mass Flow Controller), pressure sensors(MSI make Model M5256-000012 ), RTDs purifier units, heaters for regeneration etc. The storage tank of capacity about 20 liters to deliver mixed gas to RPC stack, receiver tank again of about 20 liters to receive exhaust gas from RPCs, a High Pressure is Low Pressure (HPLP) regulators (to control the flow and pressure of the mixed gas into the RPCs). At a later 
stage the HPLP regulator is changed with capillary flow resistors due to atmospheric periodic pressure variations in the laboratory.

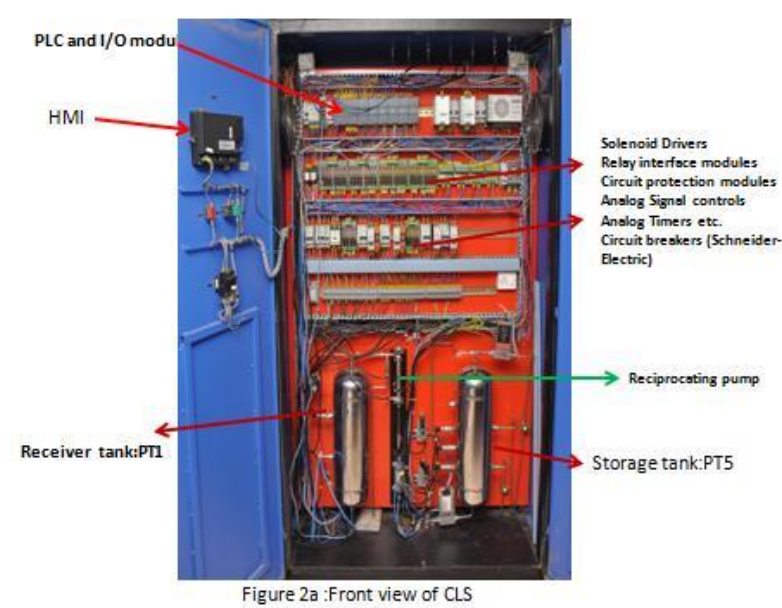

The pressure transducers have 4 to $20 \mathrm{~mA}$ current output and are used for receiver tank (PT1), radical remover tank (PT2), regeneration of molecular sieve cylinders (PT3), storage tank (PT5 and PT4), laboratory pressure monitor (PT6) and similarly the mass flow controllers MFC 1 to MFC 4 are for refilling of gas, MFC 5 for control of exhaust gas and MFC 6 for control of flow of gas into the RPCs. The relays are used to shut off the valves of MFCs by which the gas is completely stopped. The over current relays are used for the compressors, vacuum pump, electrical heater for regeneration and diaphragm pump. The timers are used to make heater ON or OFF and are used for controlling the displacement pump to start and stop and wait for particular position. A gang of solenoid valves are used for pumping of displacement pump and to control MFC supply. There are two analog inputs that are connected to the CPU from the RTDs which are monitoring temperature of gas purifier chambers. These parameters plays crucial role during recharging of molecular sieves which are used for purification of gases. The main CPU has six bit-operated digital inputs and 2 bit operated digital output (Figure 2b). It is also providing 8 digital outputs as independent channels. Digital output of CPU and Signal Module are given to solenoid driver which is used to operate solid state relays controlling heaters, air compressor, metering and vacuum pump and solenoid valves of purifier units. The LAN interface has been controlled using CPU1214C.

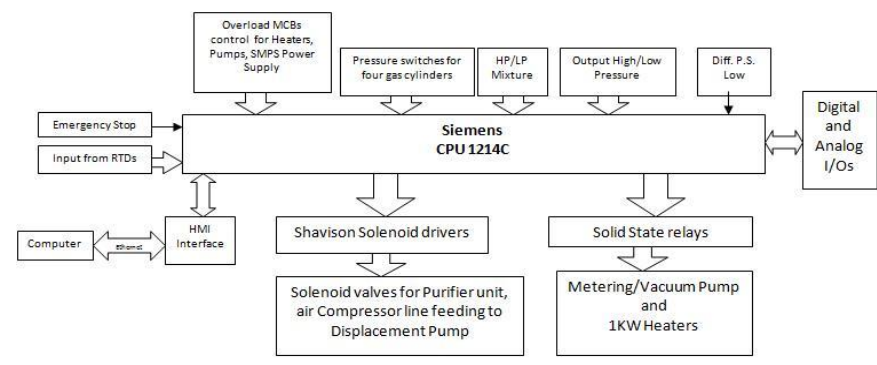

Figure 2b : PLC - Main CPU

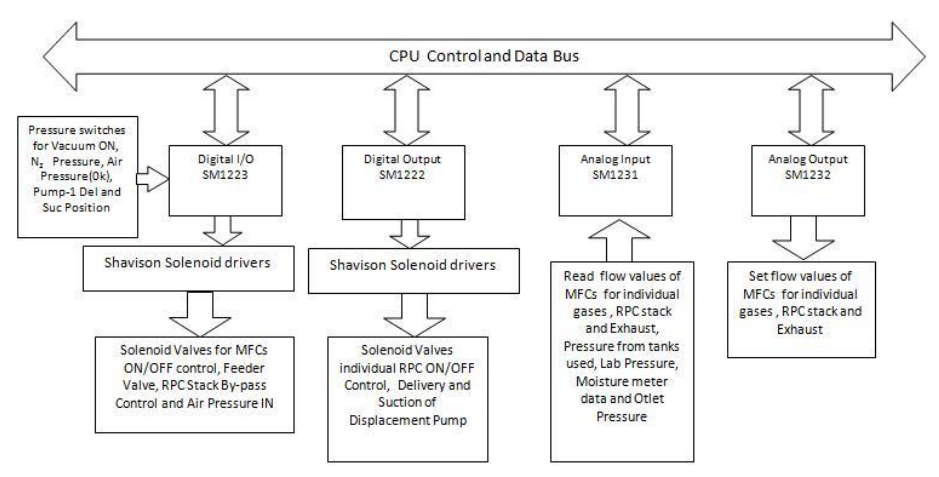

Figure 2c: Peripherals of PLC modules 
The SM1223 DC-DC Digital I/O(Figure 2c) is used to get five inputs from Nitrogen (N2) pressure input, air pressure Healthy, Pump-1 position delivery and Pump-1 position suction. Its eight outputs are connected to solenoid driver which controls the solenoids valves of MFC for on/off operation, feeder valve, air compressor and RPC stack By-pass operation. Digital output unit (SM1222 DO) used to drive 16 solenoid driver circuit (Shavison AS775) which controls the gas flow ON/OFF operation of individual RPCs. It also drives positive displacement pump. Apart from these modules two analog output modules (SM1232 AO) connected to exhaust MFC, RPCs flow MFC and individual gas MFCs to provide analog control voltage in proportional to set gas flow. One analog input module (SM1231 AI) has seven inputs from storage tank pressure (PT5), laboratory pressure(PT6), moisture meter used to check quality of gas, exhaust MFC(MFC5), RPC flow MFCs(MFC6), mixture tank-2 controller and outlet pressure. The otherSM1231 AI module is used to get the control analog voltage read values which corresponds to actual gas flow through the MFCs. The receiver tank pressure sensor (PT1) output, Purifier suction Pressure (PT2), regeneration Pressure (PT3), storage tank pressure (PT4) have been monitored through this module.

A human machine interface (HMI) is a platform which permits communication between PLC based CPU and computer interaction between users and automation equipment. It has 7 inch TFT LCD display with flash ROM of $128 \mathrm{MB}$ and $30 \mathrm{MB}$ system memory, a total of $82 \mathrm{MB}$ user memory and 16MB backup memory is available. A RS-232/485/433 communication link is also present in this model and has in build feature of saving data to pen drive without connecting to PC. It provides 8 levels of passwords to set user security to prevent improper use. A monitoring PC is connected to CLS -CPU through Ethernet via DOP e-Server data collection software (provides solution for constructing a network between office and production site). There are 28 monitoring data (pressures PT5, PT1, mass flow rates, refilling time etc.) parameters that are stored every 3 minutes in an excel sheet for offline analysis.

\subsection{Performance:}

The CLS described above with minor modification are in operation in various INO collaborating institutes. The ambient pressure cycle has great impact on the gas flow operation of CLS in absolute mode of operation, which in turn has effect on current, noise rate of the RPCs and a few RPCs were damaged [2]. A differential mode of operation has been introduced to overcome the problem.

\section{Results and Conclusion}

The Gas flow is controlled efficiently using PLC based instrumentation. All the peripherals of PCL CPU are operating as per design. The auto refill cycle of 21 days [3] has been achieved with minimum a leakage which corresponds to about 5 liters of gas. Additional of more layers shows reduction in auto-refill cycle period to 14 days, as leakages are more in connections rather than, in the CLS. This proto type CLS can be upgraded for engineering module and latter for INO-ICAL experiment with use of multiple piston system to suck the gas from the RPC detectors into the CLS with different monitoring pressure and safety levels.

\section{Acknowledgement}

We would like to thank the team at TIFR for smooth operation and maintenance of the system. We also thank the $\mathrm{M} / \mathrm{s}$ Shriram automations for software development support for the system.

\section{References}

[1]. INO Collaboration, INO Project Report, Technical Report INO/2006/01, 2006; Detailed Project Report I, Technical Report, 2007; Detailed Project Report on INO-ICAL Detector Structure II, Technical Report, 2008 (http://www.ino. tifr.res.in/ino// OpenReports/INOReport.pdf).

[2]. Effect of ambient pressure variation on closed loop gas system for India based Neutrino Observatory (INO) B Satyanarayana, G Majumder, N K Mondal, S D Kalmani, R R Shinde, and A Joshi,

[3]. http://dx.doi.org/10.1088/1748-0221/9/10/C10001

[4]. Performance of the Prototype Gas Recirculation System with built-in RGA for INO RPC system. Nuclear Instruments and Methods in Physics Research A, 661 (2012) S234-S240.

[5]. doi:10.1016/j.nima.2010.09.169 